\title{
SULLO SVILUPPO DI ALCUNE SPECIALI FUNZIONI DI UNA VARIABILE IN SERIE DI FUNZIONI SFERICHE.
}

\author{
Nota di Adolfo Viterbi (Pavia).
}

Adunanza del 23 dicembre 1906.

I. In talune questioni di Analisi applicata si presenta il problema seguente, che riflette lo sviluppo di una funzione di una variabile reale $x$ in serie di polinomi di LEGENDRE della variabile stessa:

Data una funzione $\varphi(x)$ della variabile (reale) $x$, la quale funzione sia per tutti $i$ valori di $x$, compresi fra $-\mathrm{I} e+\mathrm{I}$, rappresentabile mediante una serie di polinomi di LEgENDRE:

$$
\varphi(x)=\sum_{n=0}^{n=\infty} b_{n} X_{n},
$$

ove designi $X_{n}$ il polinomio di Legendre $\left.(\text { in } x)^{*}\right)$ d'ordine $n(n=0,1,2, \ldots)$, dedurre dai coefficienti $b_{0}, b_{1}, b_{2}, \ldots$ l'espressione dei coefficienti dello sviluppo, in serie di polinomi $X_{n}$, di una funzione della forma:

$$
f(x)=\frac{\varphi(x)}{P(x)}
$$

quando sia $P(x)$ un polinomio qualunque in $x$, soggetto all'unica condizione che nessuna delle sue radici sia compresa fra $-\mathrm{I} e+\mathrm{I}$.

Cosi $f(x)$ è senza dubbio, in generale, rappresentabile, in tutto l'intervallo accennato, mediante una serie di polinomi di Legendre. Diremo $a_{n}(n=0, \mathrm{I}, 2, \ldots) \mathrm{i}$ coefficienti dei singoli $X_{n}$ nello sviluppo cercato.

Ora, come è noto, non esiste un metodo generale il quale permetta di porre in evidenza la legge di formazione dei coefficienti dello sviluppo di $f(x)$, facendo cosi riscontro al metodo dei coefficienti indeterminati, per la deduzione dello sviluppo di $f(x)$ in serie di potenze di $x$, dall'analogo sviluppo di $\varphi(x)$. Infatti un tale metodo dovrebbe

*) E quasi superfluo ricordare, poichè cosa universalmente nota, essere : $X_{n}=\frac{I}{2^{n} \Pi_{n}} \frac{d^{n}\left(x^{2}-1\right)^{n}}{d x^{n}}$, ove si usi il simbolo II a rappresentare i fattoriali. 
evidentemente essere fondato sopra la ben nota relazione ricorrente $\left.{ }^{*}\right)$ :

$$
(n+\mathrm{I}) X_{n+1}-(2 n+\mathrm{I}) x X_{n}+n X_{n-1}=\mathrm{o},
$$

fra tre polinomi consecutivi di LEGENDRE.

(Quando, in particolare, sia: $n=0$, la (3) si riduce alla:

poichè : $X_{\mathrm{o}}=\mathrm{I}$ ).

$$
X_{1}=x
$$

Come è facile verificare, la (3) convenientemente applicata, permette di stabilire un sistema di relazioni ricorrenti, con le quali ciascuno dei coefficienti $a_{2 m+i}(i=\mathrm{I}, 2, \ldots)$ risulta espresso, mediante una combinazione lineare dei $2 m$ coefficienti [ove $m$ designi il grado di $P(x)$ ] che nello sviluppo di $f(x)$ immediatamente lo precedono e, ben s'intende, delle $b_{n}$. A sua volta ciascuno dei coefficienti: $a_{m+j}(j=0, \mathrm{x}, 2, \ldots m-\mathrm{I})$ risulta espresso mediante una combinazione lineare degli $m+j$ coefficienti $a_{n}$ che lo precedono e delle $b_{n}$.

Cosi appunto gli $m$ coefficienti: $a_{0}, a_{1}, \ldots a_{m-1}$ rimangono indeterminati. E questi si dovranno calcolare direttamente mercè la nota relazione:

$$
a_{n}=\frac{2 n+\mathrm{I}}{2} \int_{-1}^{+\mathrm{I}} \frac{\varphi(x)}{P(x)} X_{n} d x \quad(n=0,1,2, \ldots),
$$

quando si voglia determinare completamente lo sviluppo di $f(x)$, in serie di polinomi di LEGENDRE.

Però non è difficile ottenere, con opportuni artificì, le cercate espressioni dei coefficienti $a_{o}, a_{\mathrm{r}}, \ldots a_{m-\mathrm{I}}$ sotto una forma generale e semplice, espressioni che, associate alle predette relazioni ricorrenti, pure opportunamente trasformate, permettono di porre in evidenza la legge di formazione di tutte le $a_{n}$.

A stabilire appunto l'algoritmo generale che conduce allo scopo indicato, vale a dire a risolvere la questione enunciata, è dedicata la presente Nota.

2. Per fissare le idee consideriamo prima il caso particolare semplicissimo, in cui sia :

$$
P(x)=y-x,
$$

detto $y$ un numero, in modulo, $>\mathrm{I}$, mentre siano sodisfatte le condizioni (molto late del resto) $\left.{ }^{* *}\right)$ a che sia : $\frac{I}{y-x}$, suscettibile, per: $|x| \leq 1$ dello sviluppo pure ben noto:

$$
\frac{\mathrm{I}}{y-x}=\sum_{n=0}^{n=\infty}(2 n+\mathrm{I}) Y_{n} X_{n},
$$

dove designino le $Y_{n}(n=\mathrm{o}, \mathrm{I}, 2, \ldots)$ le singole funzioni sferiche di $2^{\mathrm{a}}$ specie di $y$, di grado $n$. Ciò facciamo, si perchè la discussione dettagliata di questo caso, frequentissimo a presentarsi, faciliterà poi l'esame del caso generale nel quale non sia

*) v. HeIne, Handbuch der Kugelfunctionen, vol. I, pag. 91. Siccome poi nel seguito si dovrà citare di sovente quest'opera e precisamente soltanto il suo $\mathrm{I}^{\mathrm{O}}$ volume, ci serviremo della semplice indicazione $H$, a designare appunto il volume in parola dell'opera accennata.

*) v. Heine, $\int S$ I 7 e 45 . 
$P(x)$ sottoposto ad alcuna restrizione, all'infuori di quelle enunciate, si perchè lo studio del caso in discorso dd occasione allo svolgimento di alcune particolari considerazioni.

Possiamo pertanto alla (2) dare, in questo caso particolare, la forma:

$$
(y-x) f(x)=\varphi(x) .
$$

Allora, in virtù della (3), abbiamo fra i coefficienti designati rispettivamente con $a_{n}, b_{n}$, il seguente sistema di relazioni ricorrenti $\left.{ }^{*}\right)$ :

$$
\left\{\begin{aligned}
a_{\mathrm{1}} & =3\left(y a_{\mathrm{o}}-b_{0}\right), \\
a_{n+1} & =\frac{2 n+3}{n+\mathrm{I}}\left(y a_{n}-b_{n}\right)-\frac{(2 n+3) n}{(n+\mathrm{I})(2 n-\mathrm{I})} a_{n-1} \quad(n=\mathrm{I}, 2, \ldots) .
\end{aligned}\right.
$$

Le (I) permettono di dedurre il valore di ciascun coefficiente $a_{n}$ dai due che immediatamente lo precedono, quando $n$ sia $>\mathrm{I}$, mertre naturalmente $a_{1}$ resta espresso in funzione di $a_{\mathrm{o}}$. Perció $\mathrm{i}$ coefficienti in discorso potranno risguardarsi come noti, solo quando sia noto il coefficiente $a_{0}$.

Ora, in virtù della. (4):

$$
a_{\mathrm{o}}=\frac{\mathrm{I}}{2} \int_{-1}^{+1} \frac{\varphi(x)}{y-x} d x
$$

donde, sostituendo a: $\frac{\mathrm{I}}{y-x}$ il suo sviluppo $(5)$ e, ricordando le ben note relazioni fondamentali:

$$
\left\{\begin{array}{l}
\int_{-1}^{+1} X_{p} X_{n} d x=0 \text { quando: } p \text { sia } \leqslant n, \\
\int_{-1}^{+1} X_{n}^{2} d x=\frac{2}{2 n+1},
\end{array}\right.
$$

le quali servono appunto a stabilire la (4), ricaveremo facilmente:

$$
a_{0}=\sum_{n=0}^{n=\infty} Y_{n} b_{n} \text {. }
$$

Della convergenza della serie che figura in questa relazione è assai facile accertarsi. Infatti dalle nostre premesse risulta essere la serie:

$$
\sum_{n=0}^{n=\infty} b_{n} X_{n}
$$

convergente in ugual grado nell'intervallo costituito dai valori di $x$, compresi fra - I e + I. Di più, per ogni valore di questo intervallo é:

oppure :

$$
\left|\frac{1}{y-x}\right|<\left|\frac{1}{y-1}\right|
$$

$$
\left|\frac{1}{y-x}\right|<\left|\frac{1}{y+1}\right| \text {, }
$$

*) Queste relazioni si potrebbero dedurre direttamente anche da una formula dimostrata dall'HeINE nel $\ 20$ dell'opera citata. 
a seconda rispettivamente che $y$ è $>0<0$. Da tali considerazioni risulta evidente l'equiconvergenza e perciò l'integrabilità termine a termine (nell'intervallo in discorso) della serie:

$$
\frac{\mathrm{I}}{y-x} \sum_{n=0}^{n=\infty} b_{n} X_{n}
$$

3. Procediamo ora allo studio del caso generale, nel quale sia $P(x)$ un polinomio qualunque di grado $m$.

Per porre le formule che ora stabiliremo, sotto un aspetto tipico, generale converrà considerare l'espressione di $P(x)$, mediante una combinazione lineare dei polinomi $X_{0}(=\mathrm{I}), X_{1}, \ldots X_{m}$. Sia :

$$
P(x)=\sum_{b=0}^{h=m} p_{h} X_{b}
$$

tale espressione, ove i coefficienti $p_{b}$ si calcolano in modo di per sè evidente.

Indi pongasi la (2) sotto la forma:

$$
\varphi(x)=f(x) \sum_{h=0}^{h=m} p_{h} X_{h}
$$

Di più consideriamo lo sviluppo, secondo i polinomi di Legendre, dei singoli prodotti:

$$
X_{b} X_{n} \quad\left(\begin{array}{l}
b=0,1,2, \ldots m \\
n=0,1,2, \ldots
\end{array}\right) .
$$

La reiterata applicazione della (3) mostra senz'altro (come del resto si puó dedurre, per altra via, dalla forma stessa dei polinomi $X_{n}$ ) che :

$$
X_{b} X_{n}=\sum_{q=0}^{q=b} c_{q}^{(h, n)} X_{n+b-2 q} .
$$

Il calcolo dei coefficienti, $c_{q}^{(h, n)}$ si fa assai facilmente e puo, come è chiaro essere eseguito con varii metodi diversi. Essi possono risguardarsi come parametri noti, molto più che del loro calcolo si occupó in modo particolare F. Neumann ${ }^{*}$ ).

Le (8) permettono allora di stabilire fra $\mathrm{i}$ coefficienti $a_{n}$ il seguente sistema di relazioni ricorrenti :

$$
\text { (III) }\left\{\begin{array}{l}
a_{n+m} p_{m} c_{m}^{(n+m, m)}+a_{n+m-1} p_{m-1} c_{m-1}^{(n+m-1, m-1)}+a_{n+m-2}\left(p_{m} c_{m-1}^{(n+m-2, m)}+p_{m-2} c_{m-2}^{(n+m-2, m-2)}\right)+\cdots \\
\cdots+a_{n}\left(p_{m} c_{\frac{m}{2}}^{(n m)}+p_{m-2} c_{\frac{m-2}{2}}^{(n m-2)}+\cdots+p_{0}\right) \\
\quad+a_{n-1}\left(p_{m-1} c_{\frac{m-2}{2}}^{(n-1, m-1)}+p_{m-2} c_{\frac{m-4}{2}}^{(n-1, m-3)}+\cdots+p_{1} c_{0}^{(n-1,1)}\right)+\cdots \\
\cdots+a_{n-m+2}\left(p_{m-2} c_{0}^{(n+m-2, m-2)}+p_{m} c_{1}^{(n-m, m)}\right)+a_{n-m+1} p_{m-1} c_{0}^{(n-m+1, m-1)}+a_{n-m} p_{m} c_{0}^{(n-m, m)} \\
\quad=b_{n},
\end{array}\right.
$$

quando sia $m$ un numero pari.

") v. F. Neumann, Betträge zur Theorie der Kugelfunctionen. Lipsia, 1878 (II Parte). 
Quando invece sia $m$ un numero dispari avremo il sistema di relazioni :

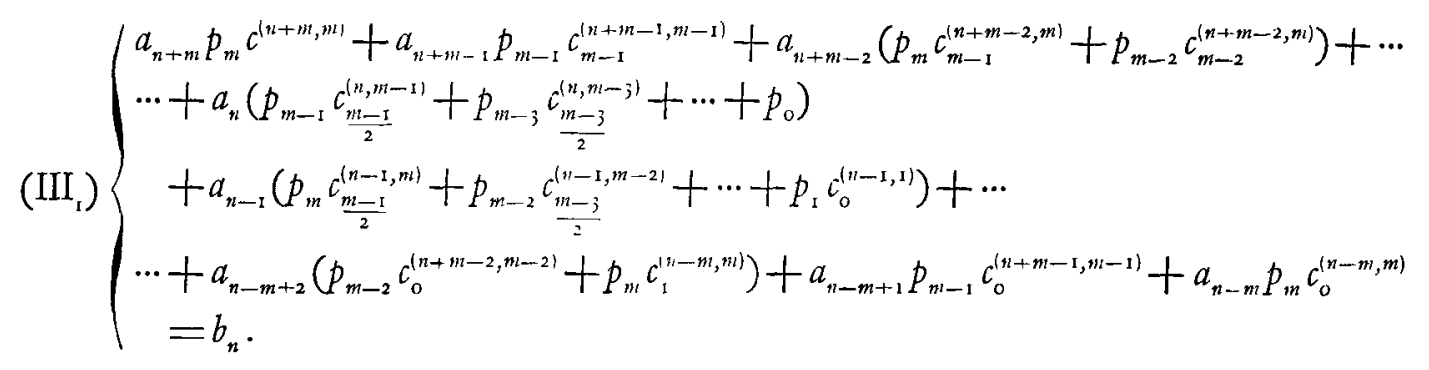

Queste relazioni si potranno ritenere valide per tutti $\mathrm{i}$ valori di $n$, compresi quelli inferiori a $m$, purchè nelle relazioni corrispondenti a questi ultimi valori si stabilisca di ritenere nulli tutti i coefficienti $a$, che risulterebbero affetti da indice negativo.

Le (III), (III $)_{i}$ mostrano all'evidenza che, come si era asserito, la completa determinazione dei coefficienti $a_{n}$ richiede che si calcolino direttamente i primi $m$ di questi. Vale a dire, una volta calcolati direttamente gli $m$ integrali definiti:

$$
\int_{-1}^{+1} \frac{\varphi(x) X_{h} d x}{P(x)} \quad(b=0,1,2, \ldots m-1)
$$

le relazioni ricorrenti, testè considerate, permetteranno di ricavare l'espressione di tutti i coefficienti $a_{n}$.

Ora, per calcolare gli $m$ integrali in parola, in guisa da ottenere formule semplici e generali ad un tempo, consideriamo lo sviluppo in serie di LegEndRE di $\frac{\mathrm{I}}{P(x)}$. A motivo delle condizioni alle quali, come fu presupposto, sodisfa $P(x)$, possiamo, in base a ben noti teoremi dovuti segnatamente al DiNI, ammettere che la reciproca di tale polinomio sia effettivamente, in tutto l'intervallo che ha per estremi - I, + I, rappresentabile mediante una serie di funzioni $X_{n}$. Sia pertanto:

$$
\frac{\mathrm{I}}{P(x)}=\sum_{n=0}^{n=\infty} s_{n} X_{n} \text {. }
$$

Da questa relazione possiamo, ricordando pure le (8), dedurre le altre:

$$
\frac{X_{h}}{P(x)}=\sum_{n=0}^{n=\infty} s_{h, n} X_{n}
$$

$$
(b=\mathrm{I}, 2, \ldots m-\mathrm{I})
$$

ove si sia posto brevemente:

$$
s_{h, n}=\sum_{q=0}^{q=h} c_{h-q}^{(n+b-2 q, h)} s_{n+h-2 q} .
$$

Ed è evidente come la (Io) rappresenti ciò, a cui si riduce la (Io'), quando: $b=o$, nel qual caso si potrd, ad ottenere maggiore simmetria nelle formule, porre:

$$
s_{0, n}=s_{n} \text {. }
$$

Si trasformino quindi gli integrali (9) col sostituire in essi a $\varphi(x), \frac{I}{P(x)}, \frac{X_{1}}{P(x)}, \ldots$, $\frac{X_{m-1}}{P(x)}$ le loro espressioni offerte rispettivamente dalle (Io), (10'). Tenendo presente 
la (4) sard allora facile ottenere le relazioni seguenti :

(IV) $\quad a_{b}=\frac{2 b+\mathrm{I}}{2} \int_{-1}^{+1} \frac{X_{b} p(x)}{P(x)} d x=(2 b+\mathrm{I}) \sum_{n=0}^{n=\infty} \frac{s_{b n} b_{n}}{2 n+\mathrm{I}} \quad(b=0, \mathrm{I}, 2, \ldots m-\mathrm{I})$.

La convergenza delle serie che figurano nei secondi membri di queste relazioni, è facile a stabilirsi, mercè considerazicni analoghe a quelle svolte alla fine del $\mathrm{n}^{\circ} \mathbf{2}$.

Le (IV) appunto, associate alle (III) [o eventualmente alle (III $\left.)_{1}\right]$ costituiscono evidentemente il cercato sistema di relazioni ricorrenti, atto a determinare $i$ coefficienti incogniti $a_{n}$, in quanto questi coeflicienti si ricavano da tale sistema, espressi mediante $\mathrm{i}$ coefficienti $p_{b}, b_{n}, s_{n}$, i quali sarebbero $\mathrm{i}$ dati del problema ed $\mathrm{i}$ coefficienti $c_{q}^{(n h)}, \mathrm{i}$ quali sono da risguardarsi come parametri fissi.

4. Quanto fu ora esposto si estende facilmente anche agli sviluppi in serie di funzioni sferiche:

$$
\frac{2^{n} \Pi(n-v) \Pi n}{\operatorname{II} 2 n}\left(\sqrt{x^{2}-\mathrm{I}}\right)^{\nu} \frac{d^{\nu} X_{n}}{d x^{\nu}}=\frac{\Pi(n-\nu)}{\Pi 2 n}\left(\sqrt{x^{2}-\mathrm{I}}\right)^{\nu} \frac{d^{n+\nu}\left(x^{2}-\mathrm{I}\right)^{n}}{d x^{n+\nu}}
$$

dove $y$ sia un numero intero fisso non $>$ di $n$. Tali sviluppi si presentano nella rappresentazione di funzioni di due variabili mediante serie di funzioni di LapLace.

Diremo brevemente $X_{n}^{(\nu)}$ le funzioni testè considerate.

Fra funzioni siffatte corrispondenti, ben s'intende, ad un medesimo indice $v$, sussistono relazioni ricorrenti ben note del tipo della (I) ${ }^{*}$ ).

Di più fra due di tali funzioni sussistono pure relazioni integrali caratteristiche di forma analoga a quelle che si hanno fra polinomi $X_{n}$.

Queste possono, come è noto, porsi sotto la forma ${ }^{* *}$ ):

$$
\int_{-1}^{+I} \frac{d^{n-\nu}\left(x^{2}-I\right)^{n}}{d x^{n-\nu}} \frac{d^{p+\nu}\left(x^{2}-I\right)^{p}}{d x^{p+y}} d x=\left\{\begin{array}{l}
(-\mathrm{I})^{\nu} \frac{2}{2 n+I} 4^{n}(\Pi n)^{2} \\
\text { oppure } \\
\text { o }
\end{array}\right.
$$

a seconda rispettivamente che $p \dot{\mathrm{e}}=n$, oppure diverso $\mathrm{da} n$.

Cosi, applicando in modo di per sè evidente tutte le relazioni accennate, sard possibile, detta $\varphi(x)$ una funzione della forma: $\left(x^{2}-1\right)^{\frac{v}{2}} \psi(x)$ [ove sia $\psi(x)$ simbolo di una funzione uniforme di $x$ ] rappresentabile (per $x$ compreso fra $-\mathbf{I}$ e $+\mathbf{I}$ ) mediante una serie della forma:

$$
\varphi(x)=\sum_{n=v}^{n=\infty} b_{n} X_{n}^{(v)}
$$

dedurre relazioni che esprimano la legge di formazione dei coefficienti $a_{n}$ dello sviluppo in serie di funzioni $X_{n}^{(\nu)}$ della funzione:

$$
f(x)=\frac{\varphi(x)}{P(x)}
$$

$[P(x)$ abbia sempre il medesimo significato sin qui attribuito a tale simbolo].

*) v. HEINE, $₫ 6_{3}$.

*) v. HeINe, $\ 62$.

Rond. Circ. Matem. Palermo, t. XXIII (1907). - Stampato il 6 febbrajo 1907. 
Cioè si potranno dedurre, in primo luogo, relazioni le quali esprimano ciascon coefficiente: $a_{n+2 m}(n=\nu, \nu+\mathrm{I}, \ldots)$ mediante i $2 m$ coefficienti che lo precedono immediatamente e i coefficienti $b_{\nu}, b_{y+1}, \ldots$ e ciascun coefficiente: $a_{\nu+2 m-i}(i=\mathrm{I}, 2, \ldots m)$ mediante i $2 m-i$ coefficienti che lo precedono e i coefficienti $b_{y}\left[a_{y}\right.$ designi il coefficiente che figura nel primo termine dello sviluppo di $f(x)$ ]. In secondo luogo si potrà dedurre direttamente dalle accennate proprietà integrali l'espressione di ciascuna delle $a_{v}, a_{y+1}, \ldots a_{v+m-1}$.

Per ottenere allora espressioni possibilmente semplici e simmetriche converrà ricercare le rappresentazioni di $P(x)$ e di: $\frac{\text { I }}{P(x)}$ rispettivamente mediante una combinazione lineare di polinomi $\frac{d^{n+\nu}\left(x^{2}-1\right)^{n}}{d x^{n+\nu}}$ ed una serie di polinomi:

$$
\frac{\mathrm{I}}{2^{n} \Pi n} \frac{d^{n-\nu}\left(x^{2}-\mathrm{I}\right)^{n}}{d x^{n-\nu}} \quad(n=\nu, \nu+\mathrm{r}, \ldots) .
$$

Ciò non presenta alcuna difficoltà sostanziale. Infatti, per $P(x)$ la rappresentazione in parola si ottiene in modo di per sé evidente.

L'accennato sviluppo di: $\frac{I}{P(x)}$ si ricaverà invece facilmente, considerando lo sviluppo, in serie di polinomi $X_{n}$, della funzione

$$
\frac{d^{\nu}}{d x^{\nu}} \frac{\mathrm{I}}{P(x)} \text {. }
$$

Anche ora la natura del polinomio $P(x)$ mostra come la funzione in parola sia effettivamente suscettibile di un tale sviluppo e come la serie di Legendre, che serve a rappresentarla, sia equiconvergente e perciò successivamente integrabile termine a termine per: $|x|<$ I.

Cosi, con semplici integrazioni (in numero di v) dello sviluppo di: $\frac{d^{\nu}}{d x^{\nu}} \frac{\mathrm{x}}{P(x)}$, in serie di polinomi $X_{n}$, si otterrà lo sviluppo cercato di: $\frac{\mathrm{I}}{P(x)}$.

Ciò premesso, sarà facile, in primo luogo determinare gli sviluppi dei prodotti:

$$
\frac{2^{n-h} \Pi(n-v) \mathrm{II} n}{\mathrm{I} \cdot 3 \ldots(2 n-\mathrm{I}) \Pi b} \frac{d^{n \pm \nu}\left(x^{2}-\mathrm{I}\right)^{n}}{d x^{n+\nu}} \frac{d^{h \pm \nu}\left(x^{2}-\mathrm{I}\right)^{b}}{d x^{h-\nu}} \quad\left(\begin{array}{l}
n=v, v+\mathrm{I}, \ldots \\
b=v, v+\mathrm{I}, \ldots v+m
\end{array}\right)
$$

mediante combinazioni lineari di polinomi:

$$
\frac{2^{n} \Pi(n-v) \Pi n}{\Pi 2 n} \frac{d^{v} X_{n}}{d x^{v}}
$$

Del pari le (II) permetteranno pure di calcolare facilmente le espressioni di $a_{v}, \ldots, a_{y+m}$, in guisa da ottenere tutti gli elementi necessari a stabilire un sistema di relazioni che, per il caso ora in esame, faccia perfetto riscontro alle (III), (IV). E dopo quanto fu detto nei $\$ \subseteq$ precedenti sarebbe superfluo soffermarsi più oltre su questo punto.

Pavia, dicembre 1906.

Adolfo Viterbi. 